\title{
Sharp estimates for the $p$-adic Hardy type operators on higher-dimensional product spaces
}

\author{
Ronghui Liu and Jiang Zhou*
}

\section{"Correspondence:}

zhoujiang@xju.edu.cn

College of Mathematics and System Science, Xinjiang University, Urumqi, 830046, People's Republic of China

\begin{abstract}
In this paper, we introduce the $p$-adic Hardy type operator and obtain its sharp bound on the $p$-adic Lebesgue product spaces. Meanwhile, an analogous result is computed for the $p$-adic Lebesgue product spaces with power weights. In addition, we characterize a sufficient and necessary condition which ensures that the weighted $p$-adic Hardy type operator is bounded on the $p$-adic Lebesgue product spaces. Furthermore, the $p$-adic weighted Hardy-Cesàro operator is also obtained.
\end{abstract}

MSC: 42B20; 42B25; 42B35

Keywords: Hardy type operator; sharp estimates; $p$-adic; product Lebesgue spaces

\section{Introduction}

$p$-adic numbers were introduced by Hensel at the end of the 19th century, they constitute an integral part of number theory, algebraic geometry, representation theory and other branches of modern mathematics (see $[1,2]$ ). However, the geometry of the space $\mathbb{Q}_{p}$ is surprisingly unlike the geometry of the space $\mathbb{R}$, in particular the Archimedean axiom is not true in $\mathbb{Q}_{p}$. Therefore the field of $p$-adic numbers has natural hierarchical structures, we refer the reader to [3-5]. In recent years, theories of functions and operators from $\mathbb{Q}_{p}^{n}$ into $\mathbb{R}$ or $\mathbb{C}$ play an important role in the $p$-adic quantum mechanics, in $p$-adic analysis. Studies of the $p$-adic Hardy operators have drawn more and more attention (for example, see [6-9]).

For a prime number $p$, let $\mathbb{Q}_{p}$ be the field of $p$-adic numbers. It is defined as the completion of the field of rational numbers $\mathbb{Q}_{p}$ with respect to the non-Archimedean $p$-adic norm $|\cdot|_{p}$. This norm is defined as follows: $|0|_{p}=0$; if any non-zero rational number $x$ is represented as $x=p^{\gamma} \frac{m}{n}$, where $\gamma$ is an integer and the integers $m, n$ are indivisible by $p$, then $|x|_{p}=p^{-\gamma}$. It is easy to see that the norm satisfies the following properties:

(i) $|x|_{p} \geq 0, \forall x \in \mathbb{Q}_{p},|x|_{p}=0 \Longleftrightarrow x=0$;

(ii) $|x y|_{p}=|x|_{p}|y|_{p}, \forall x, y \in \mathbb{Q}_{p}$;

(iii) $|x+y|_{p} \leq \max \left\{|x|_{p},|y|_{p}\right\}, \forall x, y \in \mathbb{Q}_{p}$, and when $|x|_{p} \neq|y|_{p}$, we have $|x+y|_{p}=\max \left\{|x|_{p},|y|_{p}\right\}$.

It is well known that $\mathbb{Q}_{p}$ is a typical model of non-Archimedean local fields. From the standard $p$-adic analysis, we know that any non-zero element $x$ of $\mathbb{Q}_{p}$ can be uniquely

(c) The Author(s) 2017. This article is distributed under the terms of the Creative Commons Attribution 4.0 International License (http://creativecommons.org/licenses/by/4.0/), which permits unrestricted use, distribution, and reproduction in any medium, provided you give appropriate credit to the original author(s) and the source, provide a link to the Creative Commons license, and indicate if changes were made. 
represented in the canonical series

$$
x=p^{\gamma} \sum_{j=0}^{\infty} a_{j} p^{j}, \quad \gamma=\gamma(x) \in \mathbb{Z},
$$

where $a_{j}$ are integrals, $0 \leq a_{j} \leq p-1, a_{0} \neq 0$. The series converges in the $p$-adic norm because $\left|a_{j} p^{j}\right|_{p}=p^{-j}$. Let $\mathbb{Z}_{p}=\left\{x \in \mathbb{Q}_{p}:|x|_{p} \leq 1\right\}$ be the class of all $p$-adic integrals in $\mathbb{Q}_{p}$ and denote $\mathbb{Z}_{p}^{*}=\mathbb{Z}_{p} \backslash\{0\}$.

The space $\mathbb{Q}_{p}^{n}$ denotes a vector space over $\mathbb{Q}_{p}$ which consists of all points $x=\left(x_{1}, x_{2}, \ldots\right.$, $\left.x_{n}\right)$, where $x_{i} \in \mathbb{Q}_{p}, i=1,2, \ldots, n$. The $p$-adic norm on $\mathbb{Q}_{p}^{n}$ is

$$
|x|_{p}:=\max _{1 \leq i \leq n}\left\{\left|x_{i}\right|_{p}\right\}, \quad x \in \mathbb{Q}_{p}^{n} .
$$

Denote by $B_{\gamma}(a)=\left\{x \in \mathbb{Q}_{p}^{n}:|x-a|_{p} \leq p^{\gamma}\right\}$, the ball with center at $a \in \mathbb{Q}_{p}^{n}$ and radius $p^{\gamma}$, and by $S_{\gamma}(a)=\left\{x \in \mathbb{Q}_{p}^{n}:|x-a|_{p}=p^{\gamma}\right\}$ the sphere with center at $a \in \mathbb{Q}_{p}^{n}$ and radius $p^{\gamma}$, $\gamma \in \mathbb{Z}$. It is clear that $S_{\gamma}(a)=B_{\gamma}(a) \backslash B_{\gamma-1}(a)$, and we set $B_{\gamma}(0)=B_{\gamma}$ and $S_{\gamma}(0)=S_{\gamma}$.

Since $\mathbb{Q}_{p}^{n}$ is a locally compact commutative group with respect to addition, it follows from the standard analysis that there exists a Harr measure $d x$ on $\mathbb{Q}_{p}^{n}$, which is unique up to a positive constant factor and is translation invariant. We normalize the measure $d x$ such that

$$
\int_{B_{0}(0)} d x=\left|B_{0}(0)\right|_{H}=1
$$

where $|B|_{H}$ denotes the Harr measure of a measure subset B of $\mathbb{Q}_{p}^{n}$. By simple calculation, we obtain $\left|B_{\gamma}(a)\right|=p^{\gamma n},\left|S_{\gamma}(a)\right|=p^{\gamma n}\left(1-p^{-n}\right)$.

The most fundamental averaging operator is Hardy operator defined by

$$
\mathcal{H} f(x):=\frac{1}{x} \int_{0}^{x} f(t) d t
$$

where the function $f$ is a nonnegative integrable function on $\mathbb{R}^{+}$and $x>0$. A celebrated integral inequality, due to Hardy [10], states that

$$
\|\mathcal{H} f\|_{L^{q}\left(\mathbb{R}^{+}\right)} \leq \frac{q}{q-1}\|f\|_{L^{q}\left(\mathbb{R}^{+}\right)}
$$

holds for $1<q<\infty$, and the constant $\frac{q}{q-1}$ is the best.

For the multidimensional case $n \geq 2$, generally speaking, there exist two different definitions. One is the rectangle averaging operator defined by

$$
\mathfrak{H}(f)(x):=\frac{1}{x_{1} \cdots x_{m}} \int_{0}^{x_{1}} \cdots \int_{0}^{x_{m}} f\left(t_{1}, \ldots, t_{m}\right) d t_{m} \cdots d t_{1},
$$

where the function $f$ is a nonnegative measurable function on $(0, \infty)^{n}$, and $x_{i}>0, i=$ $1,2, \ldots, m$. The boundedness of the operator $\mathfrak{H}$ is discussed in [11-14]. $\|\mathfrak{H}\|_{L^{q} \rightarrow L^{q}}$, the norm of $\mathfrak{H}$, is $\left(\frac{q}{q-1}\right)^{n}$ and obviously depends on the dimension of the space. 
The other definition is the $n$-dimensional spherical averaging operator, which was introduced by Christ and Grafakos in [15] as follows:

$$
\mathcal{H} f(x):=\frac{1}{\Omega_{n}|x|^{n}} \int_{|t| \leq|x|} f(t) d t, \quad x \in \mathbb{R}^{n} \backslash\{0\},
$$

where $\Omega_{n}$ is the volume of the unit ball in $\mathbb{R}^{n}$. The norm of $\mathcal{H}$ on $L^{q}\left(\mathbb{R}^{n}\right)$ was evaluated and found to be equal to that of the one-dimensional averaging operator. $\|\mathcal{H}\|_{L^{q} \rightarrow L^{q}}$, that is to say, does not depend on the dimension of the space.

In 2012, Fu et al. [16] defined the following $n$-dimensional $p$-adic Hardy operator:

$$
\mathcal{H}^{p} f(x):=\frac{1}{\left|B\left(0,|x|_{p}\right)\right|_{H}} \int_{|t|_{p} \leq|x|_{p}} f(t) d t, \quad x \in \mathbb{Q}_{p}^{n} \backslash\{0\}
$$

where $f$ is a nonnegative measurable function on $\mathbb{Q}_{p}^{n}, B\left(0,|x|_{p}\right)$ is a ball in $\mathbb{Q}_{p}^{n}$ with center at $0 \in \mathbb{Q}_{p}^{n}$ and radius $|x|_{p}$, and they proved the sharp estimates of the $p$-adic Hardy operator on Lebesgue spaces with power weights.

In 2013, Lu et al. [17] gave the definition of Hardy operator on higher-dimensional product spaces as follows:

$$
\mathcal{H}_{m}(f)(x):=\left(\prod_{i=1}^{m} \frac{1}{\left|B\left(0,\left|x_{i}\right|\right)\right|}\right) \int_{\left|y_{1}\right|<\left|x_{1}\right|} \ldots \int_{\left|y_{m}\right|<\left|x_{m}\right|} f\left(y_{1}, \ldots, y_{m}\right) d y_{m} \cdots d y_{1},
$$

where $f$ is a nonnegative measurable function on $\mathbb{R}^{n_{1}} \times \mathbb{R}^{n_{2}} \times \cdots \times \mathbb{R}^{n_{m}}, m \in \mathbb{N}, n_{i} \in \mathbb{N}$, $x=\left(x_{1}, x_{2}, \ldots, x_{m}\right) \in \mathbb{R}^{n_{1}} \times \mathbb{R}^{n_{2}} \times \cdots \times \mathbb{R}^{n_{m}}, x_{i} \in \mathbb{R}^{n_{i}}$ and $\prod_{i=1}^{m}\left|x_{i}\right| \neq 0$. Furthermore, the corresponding operator norm on the Lebesgue product spaces with power weights was worked out.

Next, we will introduce the definition of Hardy type operator on the higher-dimensional $p$-adic product spaces as follows and discuss the boundedness and best bound on the product of $p$-adic Lebesgue spaces.

Definition 1.1 Let $m \in \mathbb{N}, n_{i} \in \mathbb{N}, x_{i} \in \mathbb{Q}_{p}^{n_{i}}, i=1, \ldots, m$, and $f$ be a nonnegative measurable function on $\mathbb{Q}_{p}^{n_{1}} \times \mathbb{Q}_{p}^{n_{2}} \times \cdots \times \mathbb{Q}_{p}^{n_{m}}$. The $p$-adic Hardy type operator is defined by

$$
\mathcal{H}_{m}^{p}(f)(x)=\left(\prod_{i=1}^{m} \frac{1}{\left|B\left(0,\left|x_{i}\right|_{p}\right)\right|_{H}}\right) \int_{\left|y_{1}\right|_{p}<\left|x_{1}\right|_{p}} \cdots \int_{\left|y_{m}\right|_{p}<\left|x_{m}\right|_{p}} f\left(y_{1}, \ldots, y_{m}\right) d y_{m} \cdots d y_{1},
$$

where $x=\left(x_{1}, x_{2}, \ldots, x_{m}\right) \in \mathbb{Q}_{p}^{n_{1}} \times \mathbb{Q}_{p}^{n_{2}} \times \cdots \times \mathbb{Q}_{p}^{n_{m}}$ with $\prod_{i=1}^{m}\left|x_{i}\right|_{p} \neq 0$.

In 1984, Carton-Lebrun and Fosset [18] defined the weighted Hardy operator $\mathcal{H}_{\psi}$ by

$$
\mathcal{H}_{\psi}(f)(x)=\int_{0}^{1} f(t x) \psi(t) d t, \quad x \in \mathbb{R}^{n},
$$

where $\psi:[0,1] \rightarrow[0, \infty)$ is a function, and they showed the boundedness of $\mathcal{H}_{\psi}$ on Lebesgue spaces and $\mathrm{BMO}\left(\mathbb{R}^{n}\right)$ spaces. Evidently the operator $\mathcal{H}_{\psi}$ deeply depends on the nonnegative function $\psi$. For Example, when $n=1$ and $\psi(x)=1$ for $x \in[0,1]$, the operator $\mathcal{H}_{\psi}$ is just reduced to the classical Hardy operator. 
In 2006, Rim and Lee [7] defined the weighted $p$-adic Hardy operator $\mathcal{H}_{\psi}^{p}$ by

$$
\mathcal{H}_{\psi}^{p}(f)(x)=\int_{\mathbb{Z}_{p}^{*}} f(t x) \psi(t) d t, \quad x \in \mathbb{Q}_{p}^{n}
$$

where $\psi$ is a nonnegative function defined on $\mathbb{Z}_{p}^{*}$, and they gave the characterization of the function $\psi$ for which $\mathcal{H}_{\psi}^{p}$ is bounded on $L^{q}\left(\mathbb{Q}_{p}^{n}\right), 1 \leq q \leq \infty$, they also got the corresponding operator norm. Obviously, if $n=1$ and $\psi(x)=1$, then $\mathcal{H}_{\psi}^{p}$ just reduces to the $p$-adic Hardy operator $\mathcal{H}^{p}$ on $\mathbb{Q}_{p}$, which is defined by

$$
\mathcal{H}^{p} f(x):=\frac{1}{|x|_{p}} \int_{|t|_{p} \leq|x|_{p}} f(t) d t, \quad x \neq 0
$$

In 2013, Fu et al. [19] introduced the definition of weighted Hardy operator on higherdimensional product spaces as follows:

$$
\mathcal{H}_{\varphi}^{m}(f)(x)=\int_{0}^{1} \cdots \int_{0}^{1} f\left(t_{1} x_{1}, \ldots, t_{m} x_{m}\right) \varphi\left(t_{1}, \ldots, t_{m}\right) d t_{1} \cdots d t_{m}
$$

where the nonnegative function $\psi$ defined on $[0,1] \times[0,1] \times \cdots \times[0,1]$. They obtained a sufficient and necessary condition which ensures that the operator $\mathcal{H}_{\varphi}^{m}$ is bounded on the Lebesgue product spaces.

Next, we will extend the operator $\mathcal{H}_{\varphi}^{m}$ to the higher-dimensional $p$-adic product spaces.

Definition 1.2 Let $m \in \mathbb{N}, n_{i} \in \mathbb{N}, x_{i} \in \mathbb{Q}_{p}^{n_{i}}, i=1, \ldots, m$, and $f$ be a nonnegative measurable function on $\mathbb{Q}_{p}^{n_{1}} \times \mathbb{Q}_{p}^{n_{2}} \times \cdots \times \mathbb{Q}_{p}^{n_{m}}$. The $p$-adic weighted Hardy type operator is defined by

$$
\mathcal{H}_{\varphi, m}^{p}(f)(x)=\int_{\mathbb{Z}_{p}^{*}} \cdots \int_{\mathbb{Z}_{p}^{*}} f\left(t_{1} x_{1}, \ldots, t_{m} x_{m}\right) \varphi\left(t_{1}, \ldots, t_{m}\right) d t_{1} \cdots d t_{m},
$$

where $\varphi$ is a nonnegative measurable function on $\mathbb{Z}_{p}^{*} \times \mathbb{Z}_{p}^{*} \times \cdots \times \mathbb{Z}_{p}^{*}$, and $x=\left(x_{1}, x_{2}, \ldots\right.$, $\left.x_{m}\right) \in \mathbb{Q}_{p}^{n_{1}} \times \mathbb{Q}_{p}^{n_{2}} \times \cdots \times \mathbb{Q}_{p}^{n_{m}}$.

It follows from the Fubini theorem that we can easily formulate the dual operator of $\mathcal{H}_{\varphi, m}^{p}$ and denote it by $\mathcal{H}_{\varphi, m}^{p, *}$.

Definition 1.3 Let $m \in \mathbb{N}, n_{i} \in \mathbb{N}, x_{i} \in \mathbb{Q}_{p}^{n_{i}}, i=1, \ldots, m$, and $f$ be a nonnegative measurable function on $\mathbb{Q}_{p}^{n_{1}} \times \mathbb{Q}_{p}^{n_{2}} \times \cdots \times \mathbb{Q}_{p}^{n_{m}}$. The dual operator of the $p$-adic weighted Hardy type operator is defined by

$$
\mathcal{H}_{\varphi, m}^{p, *}(f)(x)=\int_{\mathbb{Z}_{p}^{*}} \cdots \int_{\mathbb{Z}_{p}^{*}} \frac{f\left(x_{1} /\left|t_{1}\right|_{p}, \ldots, x_{m} /\left|t_{m}\right|_{p}\right) \varphi\left(t_{1}, \ldots, t_{m}\right)}{\left|t_{1}\right|_{p}^{n} \cdots\left|t_{m}\right|_{p}^{n}} d t_{1} \cdots d t_{m},
$$

where $\varphi$ is a nonnegative measurable function on $\mathbb{Z}_{p}^{*} \times \mathbb{Z}_{p}^{*} \times \cdots \times \mathbb{Z}_{p}^{*}$, and $x=\left(x_{1}, x_{2}, \ldots\right.$, $\left.x_{m}\right) \in \mathbb{Q}_{p}^{n_{1}} \times \mathbb{Q}_{p}^{n_{2}} \times \cdots \times \mathbb{Q}_{p}^{n_{m}}$.

In 2014, Chuong and Hung[20] introduced the weighted Hardy-Cesàro operator, a more general form of $\mathcal{H}_{\psi}$ in the real case. 
Let $\psi:[0,1] \rightarrow[0, \infty), s:[0,1] \rightarrow \mathbb{R}$ be measurable functions. The weighted HardyCesàro operator $\mathcal{H}_{\varphi}^{s}$, associated to parameter curve $s(x, t)=s(t) x$, is defined by

$$
\mathcal{H}_{\varphi}^{s}(f)=\int_{0}^{1} f(s(t) x) \varphi(t) d t
$$

for all measurable functions $f$ on $\mathbb{R}^{n}$.

In 2014, Hung [21] considered the form of Hardy-Cesàro operator in $p$-adic analysis

$$
\mathcal{H}_{\varphi}^{p, s} f(x):=\int_{\mathbb{Z}_{p}^{*}} f(s(t) x) \varphi(t) d t
$$

where $s: \mathbb{Z}_{p}^{*} \rightarrow \mathbb{Q}_{p}$ and $\psi: \mathbb{Z}_{p}^{*} \rightarrow[0, \infty)$ are measurable functions. The author investigated the boundedness of the $p$-adic analog of the weighted Hardy-Cesàro operator on weighted Lebesgue spaces and weighted BMO spaces. In each case, the corresponding operator norms are obtained. In 2016, Chuong et al. [22] considered the boundedness of the $p$-adic weighted Hardy-Cesàro operators and their commutators on weighted functional spaces of Morrey type, and the corresponding operator norms are also computed.

Motivated by these famous results, first we will give a higher-dimensional version of the $p$-adic weighted Hardy-Cesàro operator.

Definition 1.4 Let $m \in \mathbb{N}, n_{i} \in \mathbb{N}, x_{i} \in \mathbb{Q}_{p}^{n_{i}}, i=1, \ldots, m$, and $f$ be a nonnegative measurable function on $\mathbb{Q}_{p}^{n_{1}} \times \mathbb{Q}_{p}^{n_{2}} \times \cdots \times \mathbb{Q}_{p}^{n_{m}}$. The $p$-adic weighted Hardy-Cesàro type operator is defined by

$$
\mathcal{H}_{\varphi, m}^{p, s}(f)(x)=\int_{\mathbb{Z}_{p}^{*}} \cdots \int_{\mathbb{Z}_{p}^{*}} f\left(s\left(t_{1}\right) x_{1}, \ldots, s\left(t_{m}\right) x_{m}\right) \varphi\left(t_{1}, \ldots, t_{m}\right) d t_{1} \cdots d t_{m}
$$

where $s: \mathbb{Z}_{p}^{*} \rightarrow \mathbb{Q}_{p}$ and $\varphi: \mathbb{Z}_{p}^{*} \times \mathbb{Z}_{p}^{*} \times \cdots \times \mathbb{Z}_{p}^{*} \rightarrow[0, \infty)$ are measurable functions, and $x=\left(x_{1}, x_{2}, \ldots, x_{m}\right) \in \mathbb{Q}_{p}^{n_{1}} \times \mathbb{Q}_{p}^{n_{2}} \times \cdots \times \mathbb{Q}_{p}^{n_{m}}$.

The paper is organized as follows. Section 2 is devoted to the sharp estimates of $\mathcal{H}_{m}^{p}$ on the $p$-adic Lebesgue product spaces with power weights. In Section 3, we present necessary and sufficient conditions for the boundedness of the weighted Hardy type operator and its dual operator. Furthermore, the $p$-adic weighted Hardy-Cesàro type operator also has the corresponding conclusion. In Section 4, we state explicitly the main conclusions of the research.

\section{Sharp estimates for the $\boldsymbol{p}$-adic Hardy type operator}

Theorem 2.1 Let $1<q<\infty, m \in \mathbb{N}, n_{i} \in \mathbb{N}, x_{i} \in \mathbb{Q}_{p}^{n_{i}}, i=1, \ldots$, m. Iff $\in L^{q}\left(\mathbb{Q}_{p}^{n_{1}} \times \mathbb{Q}_{p}^{n_{2}} \times \cdots \times\right.$ $\left.\mathbb{Q}_{p}^{n_{m}}\right)$, then the p-adic Hardy type operator $\mathcal{H}_{m}^{p}$ is bounded on $L^{q}\left(\mathbb{Q}_{p}^{n_{1}} \times \mathbb{Q}_{p}^{n_{2}} \times \cdots \times \mathbb{Q}_{p}^{n_{m}}\right)$, moreover, the norm of $\mathcal{H}_{m}^{p}$ can be obtained as follows:

$$
\left\|\mathcal{H}_{m}^{p}\right\|_{L^{q}\left(\mathbb{Q}_{p}^{n_{1}} \times \mathbb{Q}_{p}^{n_{2}} \times \cdots \times \mathbb{Q}_{p}^{n_{m}}\right) \rightarrow L^{q}\left(\mathbb{Q}_{p}^{n_{1}} \times \mathbb{Q}_{p}^{n_{2}} \times \cdots \times \mathbb{Q}_{p}^{n_{m}}\right)}=\prod_{i=1}^{m} \frac{1-p^{-n_{i}}}{1-p^{\frac{n_{i}}{q}-n_{i}}} .
$$

We provide the following weighted extension of this result. 
Theorem 2.2 Let $1<q<\infty, m \in \mathbb{N}, n_{i} \in \mathbb{N}, x_{i} \in \mathbb{Q}_{p}^{n_{i}}, i=1, \ldots, m$. If $\in L^{q}\left(\mathbb{Q}_{p}^{n_{1}} \times \mathbb{Q}_{p}^{n_{2}} \times\right.$ $\left.\cdots \times \mathbb{Q}_{p}^{n_{m}},|x|_{p}^{\vec{\alpha}}\right)$, where $|x|_{p}^{\vec{\alpha}}:=|x|_{p}^{\alpha_{1}} \times|x|_{p}^{\alpha_{2}} \times \cdots \times|x|_{p}^{\alpha_{m}}$ and $\alpha_{i}<(q-1) n_{i}$, then the $p$-adic Hardy type operator $\mathcal{H}_{m}^{p}$ is bounded on $L^{q}\left(\mathbb{Q}_{p}^{n_{1}} \times \mathbb{Q}_{p}^{n_{2}} \times \cdots \times \mathbb{Q}_{p}^{n_{m}},|x|_{p}^{\vec{\alpha}}\right)$, moreover, the norm of $\mathcal{H}_{m}^{p}$ can be obtained as follows:

$$
\left\|\mathcal{H}_{m}^{p}\right\|_{L}^{q\left(\mathbb{Q}_{p}^{n_{1}} \times \mathbb{Q}_{p}^{n_{2}} \times \cdots \times \mathbb{Q}_{p}^{n_{m}},|x|_{p}^{\vec{\alpha}}\right) \rightarrow L^{q}\left(\mathbb{Q}_{p}^{n_{1}} \times \mathbb{Q}_{p}^{n_{2}} \times \cdots \times \mathbb{Q}_{p}^{n_{m}},|x|_{p}^{\vec{\alpha}}\right)}=\prod_{i=1}^{m} \frac{1-p^{-n_{i}}}{1-p^{\frac{n_{i}}{q}+\frac{\alpha_{i}}{q}-n_{i}}}
$$

When $\alpha_{i}=0$, the sharp estimate of the $p$-adic Hardy type operator will be easy to get on the $p$-adic Lebesgue product spaces, so we only provide the proof of Theorem 2.1.

Proof Without loss of generality, we consider only the situation when $m=2$. Actually, a similar procedure works for all $m \in \mathbb{N}$. We set

$$
g_{f}\left(x_{1}, x_{2}\right)=\frac{1}{\left(1-p^{-n_{1}}\right)} \frac{1}{\left(1-p^{-n_{2}}\right)} \int_{\left|\xi_{1}\right| p=1} \int_{\left|\xi_{2}\right| p=1} f\left(\left|x_{1}\right|_{p}^{-1} \xi_{1},\left|x_{2}\right|_{p}^{-1} \xi_{2}\right) d \xi_{2} d \xi_{1}
$$

It is clear that $g_{f}\left(x_{1}, x_{2}\right)=g_{f}\left(\left|x_{1}\right|_{p}^{-1},\left|x_{2}\right|_{p}^{-1}\right)$, in the following we briefly call this function a radial function on the $p$-adic Lebesgue product spaces with power weights. We have

$$
\begin{aligned}
\mathcal{H}_{2}^{p}\left(g_{f}\right)\left(x_{1}, x_{2}\right)= & \frac{1}{\left|B\left(0,\left|x_{1}\right|_{p}\right)\right|_{H}} \frac{1}{\left|B\left(0,\left|x_{2}\right|_{p}\right)\right|_{H}} \int_{\left|y_{1}\right|_{p}<\left|x_{1}\right|_{p}} \int_{\left|y_{2}\right|_{p}<\left|x_{2}\right|_{p}} g_{f}\left(y_{1}, y_{2}\right) d y_{2} d y_{1} \\
= & \frac{1}{\left|B\left(0,\left|x_{1}\right|_{p}\right)\right|_{H}} \frac{1}{\left|B\left(0,\left|x_{2}\right|_{p}\right)\right|_{H}} \int_{\left|y_{1}\right|_{p}<\left|x_{1}\right|_{p}} \int_{\left|y_{2}\right|_{p}<\left|x_{2}\right|_{p}} \frac{1}{\left(1-p^{\left.-n_{1}\right)}\right.} \frac{1}{\left(1-p^{\left.-n_{2}\right)}\right.} \\
& \times \int_{\left|\xi_{1}\right|_{p}=1} \int_{\left|\xi_{2}\right|_{p=1}} f\left(\left|y_{1}\right|_{p}^{-1} \xi_{1},\left|y_{2}\right|_{p}^{-1} \xi_{2}\right) d \xi_{2} d \xi_{1} d y_{2} d y_{1} .
\end{aligned}
$$

By changing variables, $z_{i}=\left|y_{i}\right|_{p}^{-1} \xi_{i}, i=1,2$, we have

$$
\begin{aligned}
\mathcal{H}_{2}^{p}\left(g_{f}\right)\left(x_{1}, x_{2}\right)= & \frac{1}{\left(1-p^{-n_{1}}\right)} \frac{1}{\left(1-p^{-n_{2}}\right)} \frac{1}{\left|B\left(0,\left|x_{1}\right|_{p}\right)\right|_{H}} \frac{1}{\left|B\left(0,\left|x_{2}\right|_{p}\right)\right|_{H}} \\
& \times \int_{\left|y_{1}\right|_{p}<\left|x_{1}\right|_{p}} \int_{\left|y_{2}\right|_{p}<\left|x_{2}\right|_{p}} \int_{\left|z_{1}\right|_{p}=\left|y_{1}\right|_{p}} \int_{\left|z_{2}\right|_{p}=\left|y_{2}\right|_{p}} f\left(z_{1}, z_{2}\right) \\
& \times\left|y_{1}\right|_{p}^{-n_{1}}\left|y_{2}\right|_{p}^{-n_{2}} d z_{2} d z_{1} d y_{2} d y_{1} . \\
= & \frac{1}{\left(1-p^{-n_{1}}\right)} \frac{1}{\left(1-p^{\left.-n_{2}\right)}\right.} \frac{1}{\left|B\left(0,\left|x_{1}\right|_{p}\right)\right|_{H}} \frac{1}{\left|B\left(0,\left|x_{2}\right|_{p}\right)\right|_{H}} \\
& \times \int_{\left|z_{1}\right|_{p}<\left|x_{1}\right|_{p}} \int_{\left|z_{2}\right|_{p}<\left|x_{2}\right|_{p}} \int_{\left|y_{1}\right|_{p}=\left|z_{1}\right|_{p}} \int_{\left|y_{2}\right|_{p}=\left|z_{2}\right|_{p}}\left|y_{1}\right|_{p}^{-n_{1}} \\
= & \left.\frac{1}{\left|B\left(0,\left|x_{1}\right|_{p}\right)\right|_{H}} \frac{1}{\left|B\left(0,\left.\left.x_{2}\right|_{2}\right|_{p}\right)\right|_{H}} \int_{\left|z_{1}\right|_{p}<\left|x_{1}\right|_{p}} \int_{\left|z_{2}\right|_{p}<\left|x_{2}\right|_{p}} f\left(z_{1}, z_{2}\right) d z_{2}, z_{2}\right) d z_{2} d z_{1} . \\
= & \mathcal{H}_{2}^{p}(f)\left(x_{1}, x_{2}\right) .
\end{aligned}
$$


Using Hölder's inequality, we get

$$
\begin{aligned}
& \|g\|_{L q\left(\mathbb{Q}_{p}^{n_{1}} \times \mathbb{Q}_{p}^{\left.n_{2}, \vec{\alpha}\right)}\right.} \\
& =\left(\int_{\mathbb{Q}_{p}^{n_{1}}} \int_{\mathbb{Q}_{p}^{n_{2}}} \mid \frac{1}{\left(1-p^{-n_{1}}\right)} \frac{1}{\left(1-p^{-n_{2}}\right)}\right. \\
& \left.\times\left.\int_{\left|\xi_{1}\right|_{p}=1} \int_{\left|\xi_{2}\right|_{p}=1} f\left(\left|x_{1}\right|_{p}^{-1} \xi_{1},\left|x_{2}\right|_{p}^{-1} \xi_{2}\right) d \xi_{2} d \xi_{1}\right|^{q}\left|x_{1}\right|_{p}^{\alpha_{1}}\left|x_{2}\right|_{p}^{\alpha_{2}} d x_{2} d x_{1}\right)^{1 / q} \\
& \leq\left(\int_{\mathbb{Q}_{p}^{n_{1}}} \int_{\mathbb{Q}_{p}^{n_{2}}} \frac{1}{\left(1-p^{-n_{1}}\right)^{q}} \frac{1}{\left(1-p^{-n_{2}}\right)^{q}}\right. \\
& \times\left(\int_{\left|\xi_{1}\right| p=1} \int_{\left|\xi_{2}\right| p=1}\left|f\left(\left|x_{1}\right|_{p}^{-1} \xi_{1},\left|x_{2}\right|_{p}^{-1} \xi_{2}\right)\right|^{q} d \xi_{2} d \xi_{1}\right) \\
& \left.\times\left(\int_{\left|\xi_{1}\right|_{p}=1} \int_{\left|\xi_{2}\right|_{p}=1} d \xi_{2} d \xi_{1}\right)^{q-1}\left|x_{1}\right|_{p}^{\alpha_{1}}\left|x_{2}\right|_{p}^{\alpha_{2}} d x_{2} d x_{1}\right)^{1 / q} \\
& =\left(\int_{\mathbb{Q}_{p}^{n_{1}}} \int_{\mathbb{Q}_{p}^{n_{2}}} \frac{1}{\left(1-p^{-n_{1}}\right)} \frac{1}{\left(1-p^{-n_{2}}\right)}\right. \\
& \left.\times\left(\int_{\left|\xi_{1}\right|_{p}=1} \int_{\left|\xi_{2}\right|_{p}=1}\left|f\left(\left|x_{1}\right|_{p}^{-1} \xi_{1},\left|x_{2}\right|_{p}^{-1} \xi_{2}\right)\right|^{q} d \xi_{2} d \xi_{1}\right)\left|x_{1}\right|_{p}^{\alpha_{1}}\left|x_{2}\right|_{p}^{\alpha_{2}} d x_{2} d x_{1}\right)^{1 / q} \\
& =\frac{1}{\left(1-p^{-n_{1}}\right)^{1 / q}} \frac{1}{\left(1-p^{-n_{2}}\right)^{1 / q}}\left(\int _ { \mathbb { Q } _ { p } ^ { n _ { 1 } } } \int _ { \mathbb { Q } _ { p } ^ { n _ { 2 } } } \left(\int_{\left|z_{1}\right| p=\left|x_{1}\right| p} \int_{\left|z_{2}\right| p=\left|x_{2}\right| p}\right.\right. \\
& \left.\left.\times\left|f\left(z_{1}, z_{2}\right)\right|^{q}\left|x_{1}\right|_{p}^{-n_{1}}\left|x_{2}\right|_{p}^{-n_{2}} d z_{2} d z_{1}\right)\left|x_{1}\right|_{p}^{\alpha_{1}}\left|x_{2}\right|_{p}^{\alpha_{2}} d x_{2} d x_{1}\right)^{1 / q} \\
& =\frac{1}{\left(1-p^{-n_{1}}\right)^{1 / q}} \frac{1}{\left(1-p^{-n_{2}}\right)^{1 / q}}\left(\int _ { \mathbb { Q } _ { p } ^ { n _ { 1 } } } \int _ { \mathbb { Q } _ { p } ^ { n _ { 2 } } } \left(\int_{\left|x_{1}\right|_{p}=\left|z_{1}\right| p} \int_{\left|x_{2}\right|_{p}=\left|z_{2}\right|_{p}}\right.\right. \\
& \left.\left.\times\left|x_{1}\right|_{p}^{\alpha_{1}-n_{1}}\left|x_{2}\right|_{p}^{\alpha_{2}-n_{2}} d x_{2} d x_{1}\right)\left|f\left(z_{1}, z_{2}\right)\right|^{q} d z_{2} d z_{1}\right)^{1 / q} \\
& =\|f\|_{L^{q}\left(\mathbb{Q}_{p}^{n_{1}} \times \mathbb{Q}_{p}^{\left.n_{2}, \vec{\alpha}\right)} .\right.}
\end{aligned}
$$

Therefore,

$$
\frac{\left\|\mathcal{H}_{2}^{p}(f)\right\|_{L^{q}\left(\mathbb{Q}_{p}^{n_{1}} \times \mathbb{Q}_{p}^{\left.n_{2}, \vec{\alpha}\right)}\right.}}{\|f\|_{L^{q}\left(\mathbb{Q}_{p}^{n_{1}} \times \mathbb{Q}_{p}^{\left.n_{2}, \vec{\alpha}\right)}\right.}} \leq \frac{\left\|\mathcal{H}_{2}^{p}\left(g_{f}\right)\right\|_{L^{q}\left(\mathbb{Q}_{p}^{n_{1}} \times \mathbb{Q}_{p}^{\left.n_{2}, \vec{\alpha}\right)}\right.}}{\left\|g_{f}\right\|_{L^{q}\left(\mathbb{Q}_{p}^{n_{1}} \times \mathbb{Q}_{p}^{\left.n_{2}, \vec{\alpha}\right)}\right.}} .
$$

This implies the claim that if $f$ is a radial function, then we have $g_{f}=f$. This means that the norm of the operator $\mathcal{H}_{2}^{p}$ is equal to the norm that makes $\mathcal{H}_{2}^{p}$ restricted to the set of radial functions. Consequently, without loss of generality, it suffices to fulfil the proof of the theorem by assuming $f$ is a radial function.

Substituting the variable $y_{i}=\left|x_{i}\right|_{p}^{-1} z_{i}, i=1,2$, we see that $\left\|\mathcal{H}_{2}^{p}(f)\right\|_{L^{q}\left(\mathbb{Q}_{p}^{n_{1}} \times \mathbb{Q}_{p}^{n_{2}}, \vec{\alpha}\right)}$ equals

$$
\begin{gathered}
\left(\int_{\mathbb{Q}_{p}^{n_{1}}} \int_{\mathbb{Q}_{p}^{n_{2}}}\left|\mathcal{H}_{2}^{p}(f)\left(x_{1}, x_{2}\right)\right|^{q}\left|x_{1}\right|_{p}^{\alpha_{1}}\left|x_{2}\right|_{p}^{\alpha_{2}} d x_{2} d x_{1}\right)^{1 / q} \\
\quad=\left(\int_{\mathbb{Q}_{p}^{n_{1}}} \int_{\mathbb{Q}_{p}^{n_{2}}} \mid \frac{1}{\left|B\left(0,\left|x_{1}\right|_{p}\right)\right|_{H}} \frac{1}{\left|B\left(0,\left|x_{2}\right|_{p}\right)\right|_{H}}\right.
\end{gathered}
$$




$$
\begin{aligned}
& \left.\times\left.\int_{\left|y_{1}\right|_{p}<\left|x_{1}\right|_{p}} \int_{\left|y_{2}\right|_{p}<\left|x_{2}\right|_{p}} f\left(y_{1}, y_{2}\right) d y_{2} d y_{1}\right|^{q}\left|x_{1}\right|_{p}^{\alpha_{1}}\left|x_{2}\right|_{p}^{\alpha_{2}} d x_{2} d x_{1}\right)^{1 / q} \\
= & \left(\int_{\mathbb{Q}_{p}^{n_{1}}} \int_{\mathbb{Q}_{p}^{n_{2}}}\left|\int_{\left|z_{1}\right|_{p}<1} \int_{\left|z_{2}\right|_{p}<1} f\left(\left|x_{1}\right|_{p}^{-1} z_{1},\left|x_{2}\right|_{p}^{-1} z_{2}\right) d z_{2} d z_{1}\right|^{q}\left|x_{1}\right|_{p}^{\alpha_{1}}\left|x_{2}\right|_{p}^{\alpha_{2}} d x_{2} d x_{1}\right)^{1 / q} .
\end{aligned}
$$

Using the generalized Minkowski's inequality and noting that $f$ is a radial function, we have $\left\|\mathcal{H}_{2}^{p}(f)\right\|_{L^{q}\left(\mathbb{Q}_{p}^{n_{1}} \times \mathbb{Q}_{p}^{\left.n_{2}, \vec{\alpha}\right)}\right.}$ that is not greater than

$$
\begin{aligned}
& \left(\left.\int_{\mathbb{Q}_{p}^{n_{1}}} \int_{\mathbb{Q}_{p}^{n_{2}}}\left|\int_{\left|z_{1}\right|_{p}<1} \int_{\left|z_{2}\right|_{p}<1} f\left(\left|z_{1}\right|_{p}^{-1} x_{1},\left|z_{2}\right|_{p}^{-1} x_{2}\right)\right| d z_{2} d z_{1}\right|^{q}\left|x_{1}\right|_{p}^{\alpha_{1}}\left|x_{2}\right|_{p}^{\alpha_{2}} d x_{2} d x_{1}\right)^{1 / q} \\
& \quad \leq \int_{\left|z_{1}\right| p<1} \int_{\left|z_{2}\right| p<1}\left(\int_{\mathbb{Q}_{p}^{n_{1}}} \int_{\mathbb{Q}_{p}^{n_{2}}}\left|f\left(\left|z_{1}\right|_{p}^{-1} x_{1},\left|z_{2}\right|_{p}^{-1} x_{2}\right)\right|^{q}\left|x_{1}\right|_{p}^{\alpha_{1}}\left|x_{2}\right|_{p}^{\alpha_{2}} d x_{2} d x_{1}\right)^{1 / q} d z_{2} d z_{1} \\
& \quad=\int_{\left|z_{1}\right|_{p}<1} \int_{\left|z_{2}\right|_{p}<1}\left(\int_{\mathbb{Q}_{p}^{n_{1}}} \int_{\mathbb{Q}_{p}^{n_{2}}}\left|f\left(y_{1}, y_{2}\right)\right|^{q} \frac{\left|y_{1}\right|_{p}^{\alpha_{1}}}{\left|z_{1}\right|_{p}^{\alpha_{1}}} \mid \frac{\left|y_{2}\right|_{p}^{\alpha_{2}}}{\left|z_{2}\right|_{p}^{\alpha_{2}}} d y_{2} d y_{1}\right)^{1 / q}\left|z_{1}\right|_{p}^{-n_{1} / q}\left|z_{2}\right|_{p}^{-n_{2} / q} d z_{2} d z_{1} \\
& =\int_{\left|z_{1}\right|_{p}<1} \int_{\left|z_{2}\right|_{p}<1}\left|z_{1}\right|_{p}^{\frac{-n_{1}-\alpha_{1}}{q}}\left|z_{2}\right|_{p}^{\frac{-n_{2}-\alpha_{2}}{q}} d z_{2} d z_{1} \mid f \|_{L^{q}\left(\mathbb{Q}_{p}^{n_{1}} \times \mathbb{Q}_{p}^{\left.n_{2}, \vec{\alpha}\right)}\right.} \\
& \quad=\prod_{i=1}^{2} \frac{1-p^{-n_{i}}}{1-p^{\frac{n_{i}}{q}+\frac{\alpha_{i}}{q}-n_{i}}}\|f\|_{L^{q}\left(\mathbb{Q}_{p}^{n_{1}} \times \mathbb{Q}_{p}^{\left.n_{2}, \vec{\alpha}\right)} .\right.}
\end{aligned}
$$

Therefore,

$$
\left\|\mathcal{H}_{2}^{p}\right\|_{L^{q}\left(\mathbb{Q}_{p}^{n_{1}} \times \mathbb{Q}_{p}^{n_{2}},|x|_{p}^{\vec{\alpha}}\right) \rightarrow L^{q}\left(\mathbb{Q}_{p}^{n_{1}} \times \mathbb{Q}_{p}^{n_{2}},|x|_{p}^{\vec{\alpha}}\right)} \leq \prod_{i=1}^{2} \frac{1-p^{-n_{i}}}{1-p^{\frac{n_{i}}{q}+\frac{\alpha_{i}}{q}-n_{i}}} .
$$

Now let us prove that our estimate is sharp. For $0<\varepsilon<\min \left\{1,(q-1) n_{1} / q,(q-1) n_{2} / q\right\}$, we take

$$
f_{\varepsilon}\left(x_{1}, x_{2}\right)=\left|x_{1}\right|_{p}^{\frac{-n_{1}-\alpha_{1}}{q}+\varepsilon}\left|x_{2}\right|_{p}^{\frac{-n_{2}-\alpha_{2}}{q}+\varepsilon} \chi_{\left\{\left|x_{1}\right| p<1\right\}} \chi_{\left\{\left|x_{2}\right| p<1\right\}}\left(x_{1}, x_{2}\right)
$$

It follows from the elementary calculation that $\left\|f_{\varepsilon}\right\|_{L^{q}\left(\mathbb{Q}_{p}^{n_{1}} \times \mathbb{Q}_{p}^{\left.n_{2}, \vec{\alpha}\right)}\right.}$ is

$$
\begin{aligned}
& \left(\int_{\left|x_{1}\right| p<1} \int_{\left|x_{2}\right| p<1}\left|x_{1}\right|_{p}^{\left(\varepsilon-\frac{n_{1}+\alpha_{1}}{q}\right) q}\left|x_{2}\right|_{p}^{\left(\varepsilon-\frac{n_{2}+\alpha_{2}}{q}\right) q}|x|_{p}^{\vec{\alpha}} d x_{2} d x_{1}\right)^{1 / q} \\
& \quad=\left(\int_{\left|x_{1}\right| p<1}\left|x_{1}\right|_{p}^{\left(\varepsilon-\frac{n_{1}}{q}\right) q}\right)^{1 / q}\left(\int_{\left|x_{2}\right|_{p}<1}\left|x_{2}\right|_{p}^{\left(\varepsilon-\frac{n_{2}}{q}\right) q}\right)^{1 / q} \\
& =\left(\frac{1-p^{-n_{1}}}{1-p^{-q \varepsilon}}\right)^{1 / q}\left(\frac{1-p^{-n_{2}}}{1-p^{-q \varepsilon}}\right)^{1 / q} .
\end{aligned}
$$

We rewrite $\mathcal{H}_{2}^{p}\left(f_{\varepsilon}\right)$

$$
\begin{aligned}
& \mathcal{H}_{2}^{p}\left(f_{\varepsilon}\right)\left(x_{1}, x_{2}\right) \\
& \quad=\frac{1}{\left|B\left(0,\left|x_{1}\right|_{p}\right)\right|_{H}} \frac{1}{\left|B\left(0,\left|x_{2}\right|_{p}\right)\right|_{H}} \int_{\left|y_{1}\right|_{p}<\left|x_{1}\right|_{p}} \int_{\left|y_{2}\right|_{p}<\left|x_{2}\right|_{p}} f\left(y_{1}, y_{2}\right) d y_{2} d y_{1}
\end{aligned}
$$




$$
\begin{aligned}
& =\frac{1}{\left|B\left(0,\left|x_{1}\right|_{p}\right)\right|_{H}} \frac{1}{\left|B\left(0,\left|x_{2}\right|_{p}\right)\right|_{H}} \int_{\left|z_{1}\right|_{p}<1} \int_{\left|z_{2}\right|_{p}<1} f\left(z_{1}\left|x_{1}\right|_{p}^{-n_{1}}, z_{2}\left|x_{2}\right|_{p}^{-n_{2}}\right) d z_{2} d z_{1} \\
& =\frac{\left|x_{1}\right|_{p}^{\frac{-n_{1}-\alpha_{1}}{q}+\varepsilon}}{\left|B\left(0,\left|x_{1}\right|_{p}\right)\right|_{H}} \frac{\left|x_{1}\right|_{p}^{\frac{-n_{1}-\alpha_{1}}{q}+\varepsilon}}{\left|B\left(0,\left|x_{2}\right|_{p}\right)\right|_{H}} \int_{\left|z_{1}\right|_{p}<1} \int_{\left|z_{2}\right| p<1}\left|z_{1}\right|_{p}^{\frac{-n_{1}-\alpha_{1}}{q}+\varepsilon}\left|z_{2}\right|_{p}^{\frac{-n_{2}-\alpha_{2}}{q}+\varepsilon} d z_{2} d z_{1} .
\end{aligned}
$$

Thus, we estimate the norm of $\left\|\mathcal{H}_{2}^{p}\left(f_{\varepsilon}\right)\right\|_{L^{q}\left(\mathbb{Q}_{p}^{n_{1}} \times \mathbb{Q}_{p}^{\left.n_{2}, \vec{\alpha}\right)}\right.}$ as follows:

$$
\begin{aligned}
\|\left.\mathcal{H}_{2}^{p}\left(f_{\varepsilon}\right)\right|_{L^{q}\left(\mathbb{Q}_{p}^{n_{1}} \times \mathbb{Q}_{p}^{\left.n_{2}, \vec{\alpha}\right)}\right.} ^{q} & \left.\int_{\mathbb{Q}_{p}^{n_{1}}} \int_{\mathbb{Q}_{p}^{n_{2}}}\left|\int_{\left\{\left|z_{1}\right|_{p}<1,\left|z_{1}\right| p<\frac{1}{\left|x_{1}\right| p}\right\}} \int_{\left\{\left|z_{2}\right|_{p}<1,\left|z_{2}\right|_{p<}<\frac{1}{\left|x_{2}\right| p}\right\}}\right| z_{1}\right|_{p} ^{\frac{-n_{1}-\alpha_{1}}{q}+\varepsilon} \\
& \times\left.\left|z_{2}\right|_{p}^{\frac{-n_{2}-\alpha_{2}}{q}+\varepsilon} d z_{2} d z_{1}\right|_{\left|x_{1}\right|_{p}^{q \varepsilon-n_{1}-\alpha_{1}}\left|x_{2}\right|_{p}^{q \varepsilon-n_{2}-\alpha_{2}}|x|_{p}^{\vec{\alpha}} d x_{2} d x_{1}} \\
\geq & \left.\int_{\left|x_{1}\right|_{p}<1} \int_{\left|x_{2}\right|_{p}<1}\left|\int_{\left|z_{1}\right|_{p}<1} \int_{\left|z_{2}\right|_{p}<1}\right| z_{1}\right|_{p} ^{\frac{-n_{1}-\alpha_{1}}{q}+\varepsilon} \\
& \times\left.\left|z_{2}\right|_{p}^{\frac{-n_{2}-\alpha_{2}}{q}+\varepsilon} d z_{2} d z_{1}\right|^{q}\left|x_{1}\right|_{p}^{q \varepsilon-n_{1}}\left|x_{2}\right|_{p}^{q \varepsilon-n_{2}}|x|_{p}^{\vec{\alpha}} d x_{2} d x_{1} \\
= & \frac{1-p^{-n_{1}}}{1-p^{-q \varepsilon}} \frac{1-p^{-n_{2}}}{1-p^{-q \varepsilon}}\left(\int_{\left|z_{1}\right| p<1} \int_{\left|z_{2}\right| p<1}\left|z_{1}\right|_{p}^{\frac{-n_{1}-\alpha_{1}}{q}+\varepsilon}\left|z_{2}\right|_{p}^{\frac{-n_{2}-\alpha_{2}}{q}+\varepsilon} d z_{2} d z_{1}\right)^{q} \\
= & \left(\prod_{i=1}^{m} \frac{1-p^{-n_{i}}}{1-p^{\frac{n_{i}}{q}+\frac{\alpha_{i}}{q}-n_{i}-\varepsilon}}\right)^{q}\|f\|_{L^{q}\left(\mathbb{Q}_{p}^{n_{1}} \times \mathbb{Q}_{p}^{\left.n_{2}, \vec{\alpha}\right)^{*}}\right.}^{q}
\end{aligned}
$$

Therefore,

$$
\left\|\mathcal{H}_{2}^{p}\right\|_{L^{q}\left(\mathbb{Q}_{p}^{n_{1}} \times \mathbb{Q}_{p}^{n_{2}},\left.|x|\right|_{p} ^{\vec{\alpha}}\right) \rightarrow L^{q}\left(\mathbb{Q}_{p}^{n_{1}} \times \mathbb{Q}_{p}^{n_{2}},|x|_{p}^{\vec{\alpha}}\right)} \geq \prod_{i=1}^{2} \frac{1-p^{-n_{i}}}{1-p^{\frac{n_{i}}{q}+\frac{\alpha_{i}}{q}-n_{i}-\varepsilon}} .
$$

Consequently, using the definition of the norm of the operator and letting $\varepsilon \rightarrow 0$, we conclude that

$$
\left\|\mathcal{H}_{2}^{p}\right\|_{L^{q}\left(\mathbb{Q}_{p}^{n_{1}} \times \mathbb{Q}_{p}^{n_{2}},\left.|x|\right|_{p} ^{\vec{\alpha}}\right) \rightarrow L^{q}\left(\mathbb{Q}_{p}^{n_{1}} \times \mathbb{Q}_{p}^{n_{2}},|x|_{p}^{\vec{\alpha}}\right)} \geq \prod_{i=1}^{2} \frac{1-p^{-n_{i}}}{1-p^{\frac{n_{i}}{q}+\frac{\alpha_{i}}{q}-n_{i}}} .
$$

This finishes the proof of the theorem.

\section{Necessary and sufficient conditions for the boundedness of the weighted Hardy type operator}

Theorem 3.1 Let $1 \leq q \leq \infty, m \in \mathbb{N}, n_{i} \in \mathbb{N}, x_{i} \in \mathbb{Q}_{p}^{n_{i}}, i=1, \ldots, m$. $\varphi$ is a nonnegative measurable function on $\mathbb{Z}_{p}^{*} \times \mathbb{Z}_{p}^{*} \times \cdots \times \mathbb{Z}_{p}^{*}$. If $\in L^{q}\left(\mathbb{Q}_{p}^{n_{1}} \times \mathbb{Q}_{p}^{n_{2}} \times \cdots \times \mathbb{Q}_{p}^{n_{m}}\right)$, then the $p$-adic weighted Hardy type operator $\mathcal{H}_{\varphi, m}^{p}$ is bounded on $L^{q}\left(\mathbb{Q}_{p}^{n_{1}} \times \mathbb{Q}_{p}^{n_{2}} \times \cdots \times \mathbb{Q}_{p}^{n_{m}}\right)$ if and only if

$$
\int_{\mathbb{Z}_{p}^{*}} \cdots \int_{\mathbb{Z}_{p}^{*}}\left|t_{1}\right|_{p}^{-n_{1} / q} \cdots\left|t_{m}\right|_{p}^{-n_{m} / q} \varphi\left(t_{1}, \ldots, t_{m}\right) d t_{1} \cdots d t_{m}<\infty
$$

Proof Since the case $q=\infty$ is trivial, it suffices to consider the case $1 \leq q<\infty$. 
We first prove the sufficiency of Theorem 3.1. Assume

$$
\int_{\mathbb{Z}_{p}^{*}} \cdots \int_{\mathbb{Z}_{p}^{*}}\left|t_{1}\right|_{p}^{-n_{1} / q} \cdots\left|t_{m}\right|_{p}^{-n_{m} / q} \varphi\left(t_{1}, \ldots, t_{m}\right) d t_{1} \cdots d t_{m}<\infty
$$

By the generalized Minkowski's inequality, we get $\left\|\mathcal{H}_{\varphi, m}^{p}(f)\right\|_{L^{q}\left(\mathbb{Q}_{p}^{n_{1}} \times \mathbb{Q}_{p}^{n_{2}} \times \cdots \times \mathbb{Q}_{p}^{n_{m}}\right)}$ that is not greater than

$$
\int_{\mathbb{Z}_{p}^{*}} \cdots \int_{\mathbb{Z}_{p}^{*}}\left(\int_{\mathbb{Q}_{p}^{n_{1}}} \cdots \int_{\mathbb{Q}_{p}^{n_{m}}}\left|f\left(t_{1} x_{1}, \ldots, t_{m} x_{m}\right)\right|^{q} d x_{m} \cdots d x_{1}\right)^{1 / q} \varphi\left(t_{1}, \ldots, t_{m}\right) d t_{1} \cdots d t_{m}
$$

Making the change of variables $y_{i}=x_{i} t_{i}, i=1, \ldots, m$, we have

$$
\begin{aligned}
\int_{\mathbb{Z}_{p}^{*}} & \cdots \int_{\mathbb{Z}_{p}^{*}}\left(\int_{\mathbb{Q}_{p}^{n_{1}}} \cdots \int_{\mathbb{Q}_{p}^{n_{m}}}\left|f\left(y_{1}, \ldots, y_{m}\right)\right|^{q} d y_{m} \cdots d y_{1}\right)^{1 / q} \\
& \times\left|t_{1}\right|_{p}^{-n_{1} / q} \cdots\left|t_{m}\right|_{p}^{-n_{m} / q} \varphi\left(t_{1}, \ldots, t_{m}\right) d t_{1} \cdots d t_{m} \\
& \leq\|f\|_{L^{q}\left(\mathbb{Q}_{p}^{n_{1}} \times \mathbb{Q}_{p}^{n_{2}} \times \cdots \times \mathbb{Q}_{p}^{n_{m}}\right)} \int_{\mathbb{Z}_{p}^{*}} \cdots \int_{\mathbb{Z}_{p}^{*}}\left|t_{1}\right|_{p}^{-n_{1} / q} \cdots\left|t_{m}\right|_{p}^{-n_{m} / q} \varphi\left(t_{1}, \ldots, t_{m}\right) d t_{1} \cdots d t_{m} .
\end{aligned}
$$

Since the inequality

$$
\int_{\mathbb{Z}_{p}^{*}} \cdots \int_{\mathbb{Z}_{p}^{*}}\left|t_{1}\right|_{p}^{-n_{1} / q} \cdots\left|t_{m}\right|_{p}^{-n_{m} / q} \varphi\left(t_{1}, \ldots, t_{m}\right) d t_{1} \cdots d t_{m}<\infty
$$

holds, this immediately implies that the operator $\mathcal{H}_{\varphi, m}^{p}$ is bounded on $L^{q}\left(\mathbb{Q}_{p}^{n_{1}} \times \mathbb{Q}_{p}^{n_{2}} \times \cdots \times\right.$ $\left.\mathbb{Q}_{p}^{n_{m}}\right)$, and

$$
\begin{aligned}
& \left\|\mathcal{H}_{\varphi, m}^{p}\right\|_{L^{q}\left(\mathbb{Q}_{p}^{n_{1}} \times \mathbb{Q}_{p}^{n_{2}} \times \cdots \times \mathbb{Q}_{p}^{n_{m}}\right) \rightarrow L^{q}\left(\mathbb{Q}_{p}^{n_{1}} \times \mathbb{Q}_{p}^{n_{2}} \cdots \times \mathbb{Q}_{p}^{n_{m}}\right)} \\
& \quad \leq \int_{\mathbb{Z}_{p}^{*}} \cdots \int_{\mathbb{Z}_{p}^{*}}\left|t_{1}\right|_{p}^{-n_{1} / q} \cdots\left|t_{m}\right|_{p}^{-n_{m} / q} \varphi\left(t_{1}, \ldots, t_{m}\right) d t_{1} \cdots d t_{m} .
\end{aligned}
$$

This completes the proof of the sufficiency of Theorem 3.1.

Next we prove the necessary of Theorem 3.1 , if $\mathcal{H}_{\varphi, m}^{p}$ is bounded on $L^{q}\left(\mathbb{Q}_{p}^{n_{1}} \times \mathbb{Q}_{p}^{n_{2}} \times \cdots \times\right.$ $\left.\mathbb{Q}_{p}^{n_{m}}\right)$, then there exists a constant $C>0$ such that

$$
\left\|\mathcal{H}_{\varphi, m}^{p}(f)\right\|_{L^{q}\left(\mathbb{Q}_{p}^{n_{1}} \times \mathbb{Q}_{p}^{n_{2}} \times \cdots \times \mathbb{Q}_{p}^{\left.n_{m}\right)}\right.} \leq C\|f\|_{L^{q}\left(\mathbb{Q}_{p}^{n_{1}} \times \mathbb{Q}_{p}^{n_{2}} \times \cdots \times \mathbb{Q}_{p}^{n_{m}}\right)} .
$$

Now, for any $0<\varepsilon<1$ and $|\varepsilon|_{p}>1$, we take

$$
f_{i}^{\varepsilon}(x)= \begin{cases}0, & \left|x_{i}\right|_{p}<1 \\ \left|x_{i}\right|_{p}^{-\frac{n_{i}}{q}-\varepsilon}, & \left|x_{i}\right|_{p} \geq 1\end{cases}
$$

and

$$
f_{\varepsilon}\left(x_{1}, x_{2}, \ldots, x_{m}\right)=\prod_{i=1}^{m} f_{i}^{\varepsilon}(x) .
$$


Then a straightforward computation leads to

$$
\left\|f_{\varepsilon}\right\|_{L^{q}\left(\mathbb{Q}_{p}^{n_{1}} \times \mathbb{Q}_{p}^{n_{2}} \times \cdots \times \mathbb{Q}_{p}^{n_{m}}\right)}^{q}=\prod_{i=1}^{m} \frac{1-p^{-n_{i}}}{1-p^{-\varepsilon q}}
$$

Obviously, $\mathcal{H}_{\varphi, m}^{p}(f)\left(x_{1}, x_{2}, \ldots, x_{m}\right)=0$ while $\left|x_{i}\right|_{p}<1$. Moreover, we also see that, if $\left|x_{i}\right|_{p} \geq 1, \mathcal{H}_{\varphi, m}^{p}(f)\left(x_{1}, x_{2}, \ldots, x_{m}\right)$ equals

$$
\begin{aligned}
& \left|x_{1}\right|_{p}^{-\frac{n_{1}}{q}-\varepsilon} \cdots\left|x_{m}\right|_{p}^{-\frac{n_{m}}{q}-\varepsilon} \int_{\frac{1}{\left|x_{1}\right| p} \leq\left|t_{1}\right|_{p} \leq 1} \cdots \int_{\frac{1}{\left|x_{m}\right| p} \leq\left|t_{m}\right|_{p} \leq 1}\left|t_{1}\right|_{p}^{-\frac{n_{1}}{q}-\varepsilon} \cdots\left|t_{m}\right|_{p}^{-\frac{n_{m}}{q}-\varepsilon} \\
& \quad \times \varphi\left(t_{1}, \ldots, t_{m}\right) d t_{m} \cdots d t_{1} .
\end{aligned}
$$

Since the inequality

$$
\left\|\mathcal{H}_{\varphi, m}^{p}(f)\right\|_{L^{q}\left(\mathbb{Q}_{p}^{n_{1}} \times \mathbb{Q}_{p}^{n_{2}} \times \cdots \times \mathbb{Q}_{p}^{n_{m}}\right)} \leq C\|f\|_{L^{q}\left(\mathbb{Q}_{p}^{n_{1}} \times \mathbb{Q}_{p}^{n_{2}} \times \cdots \times \mathbb{Q}_{p}^{n_{m}}\right)}
$$

applies to $f_{\varepsilon}$, we have

$$
\begin{aligned}
& C^{p}\|f\|_{L^{q}\left(\mathbb{Q}_{p}^{n_{1}} \times \mathbb{Q}_{p}^{n_{2}} \times \cdots \times \mathbb{Q}_{p}^{n_{m}}\right)}^{q} \\
& \geq\left\|\mathcal{H}_{\varphi, m}^{p}\right\|_{L^{q}\left(\mathbb{Q}_{p}^{n_{1}} \times \mathbb{Q}_{p}^{n_{2}} \times \cdots \times \mathbb{Q}_{p}^{n_{m}}\right)}^{q} \\
& \geq \int_{\left|x_{1}\right|_{p} \geq 1} \cdots \int_{\left|x_{m}\right|_{p} \geq 1}\left(\left|x_{1}\right|_{p}^{-\frac{n_{1}}{q}-\varepsilon} \cdots\left|x_{m}\right|_{p}^{-\frac{n_{m}}{q}-\varepsilon}\right)^{q} \\
& \times\left(\int_{\frac{1}{\left|x_{1}\right| p} \leq\left|t_{1}\right|_{p} \leq 1} \cdots \int_{\frac{1}{\left|x_{m}\right|_{p}} \leq\left|t_{m}\right|_{p} \leq 1}\left|t_{1}\right|_{p^{-\frac{n_{1}}{q}-\varepsilon}} \cdots\left|t_{m}\right|_{p}^{-\frac{n_{m}}{q}-\varepsilon}\right. \\
& \left.\times \varphi\left(t_{1}, \ldots, t_{m}\right) d t_{m} \cdots d t_{1}\right)^{q} d x_{m} \cdots d x_{1} \\
& \geq \int_{\left|x_{1}\right|_{p} \geq|\varepsilon|_{p}} \cdots \int_{\left|x_{m}\right|_{p} \geq|\varepsilon|_{p}}\left(\left|x_{1}\right|_{p}^{-n_{1}-\varepsilon q} \cdots\left|x_{m}\right|_{p}^{-n_{m}-\varepsilon q}\right) \\
& \times\left(\int_{\frac{1}{|\varepsilon|_{p}} \leq\left|t_{1}\right|_{p} \leq 1} \cdots \int_{\frac{1}{|\varepsilon|_{p}} \leq\left|t_{m}\right|_{p} \leq 1}\left|t_{1}\right|_{p^{-\frac{n_{1}}{q}-\varepsilon}} \cdots\left|t_{m}\right|_{p}^{-\frac{n_{m}}{q}-\varepsilon}\right. \\
& \left.\times \varphi\left(t_{1}, \ldots, t_{m}\right) d t_{m} \cdots d t_{1}\right)^{q} d x_{m} \cdots d x_{1} \\
& =\left\|f_{\varepsilon}\right\|_{L^{q}\left(\mathbb{Q}_{p}^{n_{1}} \times \mathbb{Q}_{p}^{n_{2}} \times \cdots \times \mathbb{Q}_{p}^{n_{m}}\right)}^{q}|\varepsilon|_{p}^{-\varepsilon m q} \\
& \times\left(\int_{\frac{1}{|\varepsilon|_{p}} \leq\left|t_{1}\right|_{p} \leq 1} \cdots \int_{\frac{1}{|\varepsilon|_{p}} \leq\left|t_{m}\right|_{p} \leq 1}\left|t_{1}\right|_{p}^{-\frac{n_{1}}{q}-\varepsilon} \cdots\left|t_{m}\right|_{p^{-\frac{n_{m}}{q}-\varepsilon}} \varphi\left(t_{1}, \ldots, t_{m}\right) d t_{m} \cdots d t_{1}\right)^{q} .
\end{aligned}
$$

Therefore,

$$
\int_{\frac{1}{|\varepsilon| p} \leq\left|t_{1}\right|_{p} \leq 1} \cdots \int_{\frac{1}{|\varepsilon| p} \leq\left|t_{m}\right|_{p} \leq 1}\left|t_{1}\right|_{p^{-\frac{n_{1}}{q}-\varepsilon}} \cdots\left|t_{m}\right|_{p^{-\frac{n_{m}}{q}-\varepsilon}} \varphi\left(t_{1}, \ldots, t_{m}\right) d t_{m} \cdots d t_{1} \leq \frac{C}{|\varepsilon|_{p}^{\varepsilon m}}
$$


Now take $\varepsilon=p^{-k}, k=1,2, \ldots$ Then $|\varepsilon|_{p}=p^{k}>1$. Letting $k$ approach $\infty$, then $\varepsilon$ approaches 0 and $|\varepsilon|_{p}^{\varepsilon m}=p^{\frac{k m}{p^{k}}}$ approaches 1 . Then by Fatou's lemma, we obtain

$$
\int_{\mathbb{Z}_{p}^{*}} \cdots \int_{\mathbb{Z}_{p}^{*}}\left|t_{1}\right|_{p}^{-n_{1} / q} \cdots\left|t_{m}\right|_{p}^{-n_{m} / q} \varphi\left(t_{1}, \ldots, t_{m}\right) d t_{1} \cdots d t_{m}<\infty
$$

Thus, the proof of Theorem 3.1 is completed.

Since the operator $\mathcal{H}_{\varphi, m}^{p, *}$ is the dual operator of $\mathcal{H}_{\varphi, m}^{p}$, we can immediately deduce the following result.

Theorem 3.2 Let $1 \leq q<\infty, m \in \mathbb{N}, n_{i} \in \mathbb{N}$, and $\varphi$ be a nonnegative measurable function on $\mathbb{Z}_{p}^{*} \times \mathbb{Z}_{p}^{*} \times \cdots \times \mathbb{Z}_{p}^{*}, i=1, \ldots, m$.Iff $\in L^{q}\left(\mathbb{Q}_{p}^{n_{1}} \times \mathbb{Q}_{p}^{n_{2}} \times \cdots \times \mathbb{Q}_{p}^{n_{m}}\right)$, then the -adic weighted Hardy type operator $\mathcal{H}_{\varphi, m}^{p, *}$ is bounded on $L^{q}\left(\mathbb{Q}_{p}^{n_{1}} \times \mathbb{Q}_{p}^{n_{2}} \times \cdots \times \mathbb{Q}_{p}^{n_{m}}\right)$ if and only if

$$
\int_{\mathbb{Z}_{p}^{*}} \cdots \int_{\mathbb{Z}_{p}^{*}}\left|t_{1}\right|_{p}^{-\frac{(q-1) n_{1}}{q}} \cdots\left|t_{m}\right|_{p}^{-\frac{(q-1) n_{m}}{q}} \varphi\left(t_{1}, \ldots, t_{m}\right) d t_{1} \cdots d t_{m}<\infty
$$

Remark 3.1 Using methods similar to Theorem 3.1, we can easily see the proof of Theorem 3.2. So we omit the details of the proof. In particular, if $\varphi=1$, we know that the operator $\mathcal{H}_{\varphi, m}^{p}$ is not bounded on $L^{1}\left(\mathbb{Q}_{p}^{n_{1}} \times \mathbb{Q}_{p}^{n_{2}} \times \cdots \times \mathbb{Q}_{p}^{n_{m}}\right)$. In the same way, when $\varphi=1$, we can also deduce that $\mathcal{H}_{\varphi, m}^{p, *}$ is not bounded on $L^{\infty}\left(\mathbb{Q}_{p}^{n_{1}} \times \mathbb{Q}_{p}^{n_{2}} \times \cdots \times \mathbb{Q}_{p}^{n_{m}}\right)$.

We note that in [23], the authors proved the sharp bounds of a weighted multilinear Hardy-Cesàro operator on the product of Lebesgue spaces and central Morrey spaces. They also proved sufficient and necessary conditions of the weight functions so that the commutators of weighted multilinear Hardy-Cesàro operator with symbols in central BMO spaces.

Inspired by the paper, we will consider sufficient and necessary conditions of the weighted Hardy-Cesàro type operator $\mathcal{H}_{\varphi, m}^{p, s}$ on the $p$-adic Lebesgue product spaces.

Theorem 3.3 Suppose $1 \leq q<\infty, m \in \mathbb{N}, n_{i} \in \mathbb{N}$. Let $s: \mathbb{Z}_{p}^{*} \rightarrow \mathbb{Q}_{p}$ be a measurable function such that $\left|s\left(t_{i}\right)\right|_{p} \geq\left|t_{i}\right|_{p}^{\beta}$ for some real $\beta$ and almost everywhere $t_{i} \in \mathbb{Z}_{p}^{*}$. If $f \in L^{q}\left(\mathbb{Q}_{p}^{n_{1}} \times\right.$ $\left.\mathbb{Q}_{p}^{n_{2}} \times \cdots \times \mathbb{Q}_{p}^{n_{m}}\right)$, then the $p$-adic weighted Hardy-Cesàro type operator $\mathcal{H}_{\varphi, m}^{p, s}$ is bounded on $L^{q}\left(\mathbb{Q}_{p}^{n_{1}} \times \mathbb{Q}_{p}^{n_{2}} \times \cdots \times \mathbb{Q}_{p}^{n_{m}}\right)$ if and only if

$$
\int_{\mathbb{Z}_{p}^{*}} \cdots \int_{\mathbb{Z}_{p}^{*}}\left|s\left(t_{1}\right)\right|_{p}^{-n_{1} / q} \cdots\left|s\left(t_{m}\right)\right|_{p}^{-n_{m} / q} \varphi\left(t_{1}, \ldots, t_{m}\right) d t_{1} \cdots d t_{m}<\infty
$$

Remark 3.2 Noting that Theorem 3.3 is a more general result than the above two theorems, the operator $\mathcal{H}_{\varphi, m}^{p, s}$ is reduced to the weighted Hardy type operator and its dual operator if $s(t)$ is equal to some suitable functions. The sufficiency of Theorem 3.3 can be obtained easily by the generalized Minkowski inequality and a $p$-adic change of variables. It is worth pointing out that we need to make some appropriate modifications for the necessity. Applying this condition $|s(t)|_{p} \geq|t|_{p}^{\beta}$, then the area of $s(t)$ is converted into the relevant area of $t$, so it is easy to get the desired results by adopting the same method as Theorem 3.1. 


\section{Conclusions}

In the present study, we introduced a class of $p$-adic Hardy type operator and considered the problem of finding a bound for the norm of it from $p$-adic Lebesgue product spaces with power weights to itself. Moreover, we characterized a sufficient and necessary condition which ensures that the weighted $p$-adic Hardy type operator is bounded on the $p$-adic Lebesgue product spaces. In addition, we also extended the results to the $p$-adic weighted Hardy-Cesàro operator.

\section{Acknowledgements}

The authors would like to express their thanks to the referees for valuable advice regarding previous version of this paper. The research was supported by NNSF of China (Grant No. 11661075).

\section{Competing interests}

The authors declare that they have no competing interests.

\section{Authors' contributions}

All authors contributed equality and significantly in writing this paper. All authors read and approved the final manuscript.

\section{Publisher's Note}

Springer Nature remains neutral with regard to jurisdictional claims in published maps and institutional affiliations.

Received: 12 April 2017 Accepted: 29 August 2017 Published online: 12 September 2017

\section{References}

1. Albeverio, S, Karwoski, W: A random walk on p-adics: the generator and its spectrum. Stoch. Process. Appl. 53, 1-22 (1994)

2. Khrennikov, A: p-Adic Valued Distributions in Mathematical Physics. Kluwer Academic, Dordrecht (1994)

3. Taibleson, M: Fourier Analysis on Local Fields. Princeton University Press, Princeton (1975)

4. Vladimirov, VS, Volovich, IV, Zelenov, El: p-Adic Analysis and Mathematical Physics. World Scientific, Singapore (1994)

5. Vladimirov, VS, Volovich, IV: p-Adic quantum mechanics. Commun. Math. Phys. 123(4), 659-676 (1989)

6. Rim, YC: Carleson measures and the BMO space on the $p$-adic vector space. Math. Z. 282(9), 1470-1477 (2009)

7. Rim, KS, Lee, J: Estimates of weighted Hardy-Littlewood averages on the $p$-adic vector space. J. Math. Anal. Appl. 324(2), 1470-1477 (2006)

8. Wu, QY, Fu, ZW: Sharp estimates of $m$-linear $p$-adic Hardy and Hardy-Littlewood-Pólya operators. J. Appl. Math. 2011(1), 137-150 (2011)

9. Wu, QY, Fu, ZW: Weighted $p$-adic Hardy operators and their commutators on $p$-adic central Morrey spaces. Bull. Malays. Math. Sci. Soc. 40(2), 635-654 (2017)

10. Hardy, GH: Note on a theorem of Hilbert. Math. Z. 6, 314-317 (1920)

11. Bényi, Á, Oh, C: Best constants for certain multilinear integral operators. J. Inequal. Appl. 2006(1), Article ID 28582 (2006)

12. Wang, SM, Lu, SZ, Yan, DY: Explicit constants for Hardy's inequality with power weight on $n$-dimensional product spaces. Sci. China Math. 55(12), 2469-2480 (2012)

13. Muckenhoupt, B: Weighted norm inequalities for classical operators. Proc. Symp. Pure Math. 35, 69-83 (1979)

14. Pachpatte, BG: On multivariate Hardy type inequalities. An. Știinţ. Univ. 'Al.I. Cuza' laşi, Mat. 38, 355-361 (1992)

15. Christ, M, Grafakos, L: Best constants for two nonconvolution inequalities. Proc. Am. Math. Soc. 123(6), 1687-1693 (1995)

16. Fu, ZW, Wu, QY, Lu, SZ: Sharp estimates of $p$-adic Hardy and Hardy-Littlewood-Pólya operators. Acta Math. Sin. 29(1), 137-150 (2012)

17. Lu, SZ, Yan, DY, Zhao, FY: Sharp bounds for Hardy type operators on higher-dimensional product spaces. J. Inequal. Appl. 2013(1), Article ID 148 (2013)

18. Carton-Lebrun, C, Fosset, M: Moyennes et quotients de Taylor dans BMO. Bull. Soc. R. Sci. Liège 53(2), 85-87 (1984)

19. Fu, ZW, Gong, SL, Lu, SZ, Yuan, W: Weighted multilinear Hardy operators and commutators. Forum Math. 27(5), 2825-2851 (2015)

20. Chuong, NM, Hung, HD: Bounds of weighted Hardy-Cesáro operators on weighted Lebesgue and BMO spaces. Integral Transforms Spec. Funct. 25(9), 697-710 (2014)

21. Hung, HD: The $p$-adic weighted Hardy-Cesáro operator and an application to discrete Hardy inequalities. J. Math. Anal. Appl. 409(2), 868-879 (2014)

22. Chuong, NM, Hung, HD, Hong, NT: Bounds of $p$-adic weighted Hardy-Cesáro operators and their commutators on p-adic weighted spaces of Morrey types. P-Adic Numb. Ultr. Anal. Appl. 8, 30-43 (2016)

23. Hung, HD, Ky, LD: New weighted multilinear operators and commutators of Hardy-Cesáro type. Acta Math. Sci. Ser. B Engl. Ed. 35(6), 1411-1425 (2015) 